\title{
Astragalus affects fecal microbial composition of young hens as determined by $16 \mathrm{~S}$ rRNA sequencing
}

Hongxing Qiao ${ }^{1,2,3}$, Liheng Zhang ${ }^{1,2,3}$, Hongtao Shi $i^{1,2,3}$, Yuzhen Song ${ }^{1,2,3}$ and Chuanzhou Bian ${ }^{1,2,3^{*}}$ (D)

\begin{abstract}
The gut microbiota play important roles in the degradation of chemical compounds of herbal medicines (HMs). However, little information regarding the interplay between HMs and the gut microbiota is available. Thus, the aim of this study was to investigate the composition of the fecal microbiota of young (age, 11 weeks) hens fed a conventional diet containing a crude Astragalus (0.5\%) additive for 21 days (group A) vs. controls (group B) that were fed only conventional feed. The fecal contents of 14-week-old hens were collected for DNA extraction, and then the $\mathrm{V} 3$ and V4 hyper-variable regions of the $16 \mathrm{~S}$ rRNA gene were amplified and analyzed using high-throughput sequencing technology. A distinctive difference in microbial diversity was observed between the two groups. The microbial composition of hens fed a diet supplemented with Astragalus was greater than that of the control group. At the genus level, Lactobacillus was more abundant in group A than group B $(p<0.05)$. Importantly, this study is the first to report the observation of a novel Romboutsia sp. in the feces of hens. However, Romboutsia was less abundant in group $A$ than group B (17.94 vs. 33.98\%, respectively, $p<0.05)$. The microbial community differed significantly between the two groups at the genus level, suggesting that Astragalus modulates the composition of the fecal microbiota. Based on these differences, these findings provide fresh insights into the application of Astragalus in the poultry industry, as well as a better understanding of the interplay between HMs and the gut microbiota.
\end{abstract}

Keywords: Herbal medicine, Microbiota, NGS, Lactobacillus, Romboutsia

\section{Introduction}

Poultry meat and eggs are common nutritious and healthy sources of animal protein for human consumption. However, the accumulation of antibiotic residues in chickens and eggs, and the subsequent prevalence of drug-resistant pathogens have received attention worldwide (Wang et al. 2017b). The use of antibiotics can result in gut dysbiosis, diarrhea, and host immune dysregulation (Willing et al. 2011), which results in reduced growth and production (Gao et al. 2017). Recent studies have shown that the composition of the gut microbiota affects various physiological functions of the host, such

\footnotetext{
*Correspondence: chuanzhou-bian@126.com

${ }^{1}$ College of Veterinary Medicine, Henan University of Animal Husbandry and Economy, Longzihu North road No.6, Zhengzhou 450046, Henan, People's Republic of China

Full list of author information is available at the end of the article
}

as nutrient utilization, gut epithelium nourishment, and the development and activity of the gut immune system (Ismail et al. 2009 and Hill et al. 2010).

Herbal medicines (HMs), such as botanical medicines and phytomedicines, have contributed significantly to human and animal health when used to treat disease (Qiu 2007). Moreover, a recent study focusing on the interplay between HMs and the gut microbiota showed that the chemical components of HMs were metabolized by the gut microbiota to generate metabolites that have different bioactivities, which can also mediate the composition of the gut microbiota, restore host homeostasis, and ameliorate gut dysfunction (Chen et al. 2016). A multitude of studies supports the use of HMs, herbal formulas, and phytochemicals, such as Gegen Qinlian Decoction formula (Xu et al. 2015), Qushi Huayu Decotion formula (Yang et al. 2017), Rhizoma coptidis (Xie et al. 2011), and 
quercetin (Li et al. 2016), to modulate the gut microbiota. Therefore, the gut microbiota plays an important role in the therapeutic efficacy of HMs (Xu et al. 2017).

In China, Astragalus is a common HM that contains polysaccharides, saponins, flavonoids, anthraquinones, alkaloids, amino acids, $\beta$-sitosterol, and metallic elements (Ibrahim et al. 2013; Li et al. 2009). The major polysaccharides present in Astragalus are mannose, D-glucose, D-galactose, xylose, and L-arabinose (Kallon et al. 2013). Our previous studies showed that Astragalus fermented by Bacillus subtilis using liquid fermentation technology is beneficial to Astragalus polysaccharide production (Qiao et al. 2017). The major flavonoids of Astragalus are 3-O- $\beta$-D-gluside, $2^{\prime}$-hydroxy- $3^{\prime}, 4^{\prime}$ dimethoxyisoflavane7-O- $\beta$-D-gluside, $7,3^{\prime}$-dihydroxy- $4^{\prime}$ methoxyisoflavone,7,3-dimer-capto-4,1-methoxyisoflavone, 3-dimercapto-7,4,1-methoxyisoflavone, and kumatakenin (Lv et al. 2011; Xiao et al. 2004). Moreover, Astragalus has anti-inflammatory (Kim et al. 2013), immunostimulatory (Qin et al. 2012), antioxidative (Kim and Yang 2005), and antiviral activities (Sanpha et al. 2013). Therefore, Astragalus is used as an additive to the feed of livestock and poultry.

High-throughput, next-generation sequencing (NGS) based on 16S rRNA gene amplicons has the advantage of exploring the more complex aspects of the microbiota in animals. Moreover, studies have reported the composition of the gut microbiota of broiler chickens (Mohd Shaufi et al. 2015), Dagu chickens (Xu et al. 2016), Naked Neck chickens (Park et al. 2016), and egg-laying hens (Videnska et al. 2014). The major phyla in fecal samples of laying hens include Firmicutes (58.8\%), Bacteroidetes (22.1\%), Proteobacteria (16.9\%), Actinobacteria $(0.6 \%)$, and Fusobacteria (1.4\%) (Videnska et al. 2014). Some functional feed additives, such as prebiotics (Rastall and Gibson 2015), probiotics (Gao et al. 2017), phytase (Borda-Molina et al. 2016), and fermented Ginkgo leaves (Zhang et al. 2015), are reported to maintain microbial populations and support the health of the host. NGS has facilitated in-depth studies on the effect of HM on gut microbiota. However, few studies have investigated the microbiota of young hens fed a diet containing Astragalus. The results of our previous study showed that both fermented and unfermented Astragalus can modulate the intestinal microbiota of broilers (unpublished observations). In the present study, we hypothesized that Astragalus supplementation may alter the composition of the fecal microbiota of young laying hens. Using the Illumina MiSeq platform, we attempted to determine whether Astragalus is associated with changes in the gut microbiota.

\section{Materials and methods} Birds and management

A total of $60 \mathrm{Hy}$-Line Brown hens (mean age, 11 weeks; mean body weight, $1.32 \mathrm{~kg}$ ) were assigned randomly to two groups (A and B) of 30 pullets each. For each group, 10 pullets were housed in separate pens $\left(\right.$ area, $>3000 \mathrm{~cm}^{2}$ ) with ad libitum access to feed and water, a constant room temperature at $16-25^{\circ} \mathrm{C}$, and a $14: 10$-h light:dark cycle. The hens were checked twice daily by trained staff during the entire 3-week experimental period. All procedures were performed in accordance with the guidelines of the Ministry of Agriculture of China. Hens in group $B$ served as the control group and were fed only conventional feed (Table 1), while those in group A were fed conventional feed supplemented with a crude Astragalus $(0.5 \%)$ additive throughout the experimental period. The animal experiments were conducted in accordance with the Guidelines for the Care and Use of Experimental Animals established and approved by the Laboratory Animal Management Committee of Henan University of Animal Husbandry and Economy.

\section{Preparation of Astragalus}

Astragalus, the dried root of Astragalus membranaceus (Fisch.) Bge. var. mongholicus was obtained from a Chinese medicine market (Minxian, Gansu, China) and verified by Dr. Zhang Jing Yu (Henan University of Traditional Chinese Medicine, Zhengzhou, Henan, China). The contents of Astragalus polysaccharides, astragalosides, and total flavonoids were 23.420, 0.170, and $4.755 \mathrm{mg} / \mathrm{g}$, respectively. Then, the Astragalus root was ground into powder, sieved through a 100-mesh filter, and stored at $25^{\circ} \mathrm{C}$ for further use.

\section{Sample collection and DNA extraction}

A total of 27 fecal samples (13 from group A and 14 from group $B$ ) were chosen randomly from the hens at the age of 14 weeks. The fecal samples were immediately stored at $-20{ }^{\circ} \mathrm{C}$ until DNA extraction. DNA was isolated from $200 \mathrm{mg}$ of feces from each hen using a commercial DNA extraction kit (Tiangen Biotech Corporation, Beijing, China) and quantified using a Qubit 2.0 fluorometer (Invitrogen Corporation, Carlsbad, CA, USA). The quality

\begin{tabular}{lll}
$\begin{array}{l}\text { Table } 1 \text { Composition of the basic diet } \\
\text { weight) }\end{array}$ & Control group & Treatment group \\
\hline Ingredient & 66.70 & 66.70 \\
\hline Corn & 21.70 & 21.20 \\
Soybean meal & 6.20 & 6.20 \\
Wheat bran & 2.00 & 2.00 \\
Fish meal & 1.10 & 1.10 \\
Dicalcium phosphate & 1.30 & 1.30 \\
Limestone & 1.00 & 1.00 \\
Premix & 0 & 0.50 \\
Astragalus & 100 & 100 \\
Total & & \\
\hline
\end{tabular}


of the extracted DNA was assessed by $0.8 \%$ agarose gel electrophoresis and spectrophotometry (optical density at $260 / 280 \mathrm{~nm}$ ). All extracted DNA samples were stored at $-20{ }^{\circ} \mathrm{C}$ until further analysis.

\section{Library preparation and Illumina MiSeq sequencing}

NGS library preparations and Illumina MiSeq sequencing were conducted by GENEWIZ, Inc. (Suzhou, China). For the library preparation, a library sequence of the $\mathrm{V} 3$ and V4 regions of $16 \mathrm{~S}$ rRNA was constructed using a 10-ng DNA aliquot isolated from each fecal sample. The V3 and V4 regions were amplified by polymerase chain reaction (PCR) using the following primer pair: forward 5'-CCT ACG GRR BGC ASC AGK VRV GAA T- $3^{\prime}$ and reverse $5^{\prime}-$ GGA CTA CNY VGG GTW TCT AAT CC $-3^{\prime}$. The first-round PCR products were used as templates for a second round of amplicon enrichment by PCR $\left(94{ }^{\circ} \mathrm{C}\right.$ for $3 \mathrm{~min}$, followed by 24 cycles at $94{ }^{\circ} \mathrm{C}$ for $5 \mathrm{~s}, 57{ }^{\circ} \mathrm{C}$ for $90 \mathrm{~s}$, and $72{ }^{\circ} \mathrm{C}$ for $10 \mathrm{~s}$, and a final extension at $72{ }^{\circ} \mathrm{C}$ for $5 \mathrm{~min}$ ). At the same time, indexed adapters were added to the ends of the $16 \mathrm{~S}$ rDNA amplicons to generate indexed libraries that were ready for downstream NGS on the MiSeq platform. DNA libraries were validated using an Agilent 2100 Bioanalyzer (Agilent Technologies, Palo Alto, CA, USA) and quantified with a Qubit 2.0 Fluorometer. DNA libraries were multiplexed and loaded on an Illumina MiSeq instrument (Illumina, San Diego, CA, USA) according to the manufacturer's instructions. Sequencing was performed using a $2 \times 300$ paired-end configuration and image analysis and base calling were conducted with the MiSeq Control Software embedded in the MiSeq instrument. The library used in this study was constructed from a total of 27 DNA samples. The sequences generated in this study have been deposited in the Sequence Read Archive of the National Center for Biotechnology Information (https://www.ncbi. nlm.nih.gov/biosample) under the accession numbers SAMN07135769-7135795.

\section{Statistical analysis}

The QIIME (Quantitative Insights Into Microbial Ecology; ver. 1.9.1) data analysis package was used for $16 \mathrm{~S}$ rRNA data analysis. The forward and reverse reads were joined and assigned to samples based on barcodes, and truncated by cutting off the barcode and primer sequences. Quality filtering of the joined sequences was performed and sequences that did not fulfill the following criteria were discarded: sequence length $<200 \mathrm{bp}$, no ambiguous bases, and mean quality score $\geq 20$. Then, the sequences were compared with a reference database [the Ribosomal Database Project (RDP) Gold database, 2.2] using the UCHIME algorithm to detect chimeric sequences, which were subsequently removed.
The effective sequences were used in the final analysis. Sequences were grouped into operational taxonomic units (OTUs) using the clustering program VSEARCH (1.9.6) against the SILVA 119 database that was pre-clustered at a $97 \%$ sequence identity. The RDP classifier was used to assign a taxonomic category to all the OTUs at a confidence threshold of 0.8. Taxonomic categories at the species level were predicted with the RDP classifier and the SILVA 119 database.

Sequences were rarefied prior to calculating alpha and beta diversity statistics. Alpha diversity indices were calculated in QIIME from rarefied samples using the Shannon and Simpson indexes for diversity, and the Chao1 and ACE indexes for richness. Statistical analysis was performed with the Student's $t$ test using IBM SPSS Statistics 24 (IBM Corp., Armonk, NY, USA) and the results are presented as the mean \pm standard deviation. Beta diversity was calculated using weighted and unweighted UniFrac distances, and principal coordinate analysis (PCoA) was performed. An arithmetic mean phylogenetic tree was constructed from the beta diversity distance matrix with an unweighted pair group method. The Student's $t$-test was employed for analysis of the relative abundance at the phylum and genus levels. A probability $(p)$ value of $<0.05$ was considered statistically significant. Differences between the two groups were compared using STAMP (2.1.3) analysis.

\section{Results}

\section{OTU clustering and annotation}

A total of $60 \mathrm{Hy}$-Line Brown hens at the age of 11 weeks were assigned randomly to group A or B. Hens in group $B$ were fed only conventional feed, while those in group A were fed conventional feed supplemented with $0.5 \%$ crude Astragalus throughout the entire experimental period. Twenty-seven fecal samples (14 from group A and 13 from group B) were collected. A total of $2,945,166$ sequence reads with a median length $450 \mathrm{bp}$ were obtained from all fecal samples. The sequences were further clustered into 317 OTUs using a 97\% similarity cut-off. A clustering analysis of 30 OTUs with the highest default abundances showed both similarities and differences between the samples (Fig. 1). For example, the abundance of OTU1 was similar across 27 samples, while there were no differences in OTU2, 3, 4, and 5, except for individual samples (B5, B7, B13). There were some similarities in the OTUs among samples A7, A12, A13, B1, B2, B11, and B12, and dissimilarities among the rest. Using an abundance-based coverage estimator, the Chao1, Simpson's index Ds, and Shannon's index H' identified differences in species richness between the groups (Table 2). The Simpson index of group A was significantly higher $(p<0.05)$ than that of group $\mathrm{B}$, while there were no 


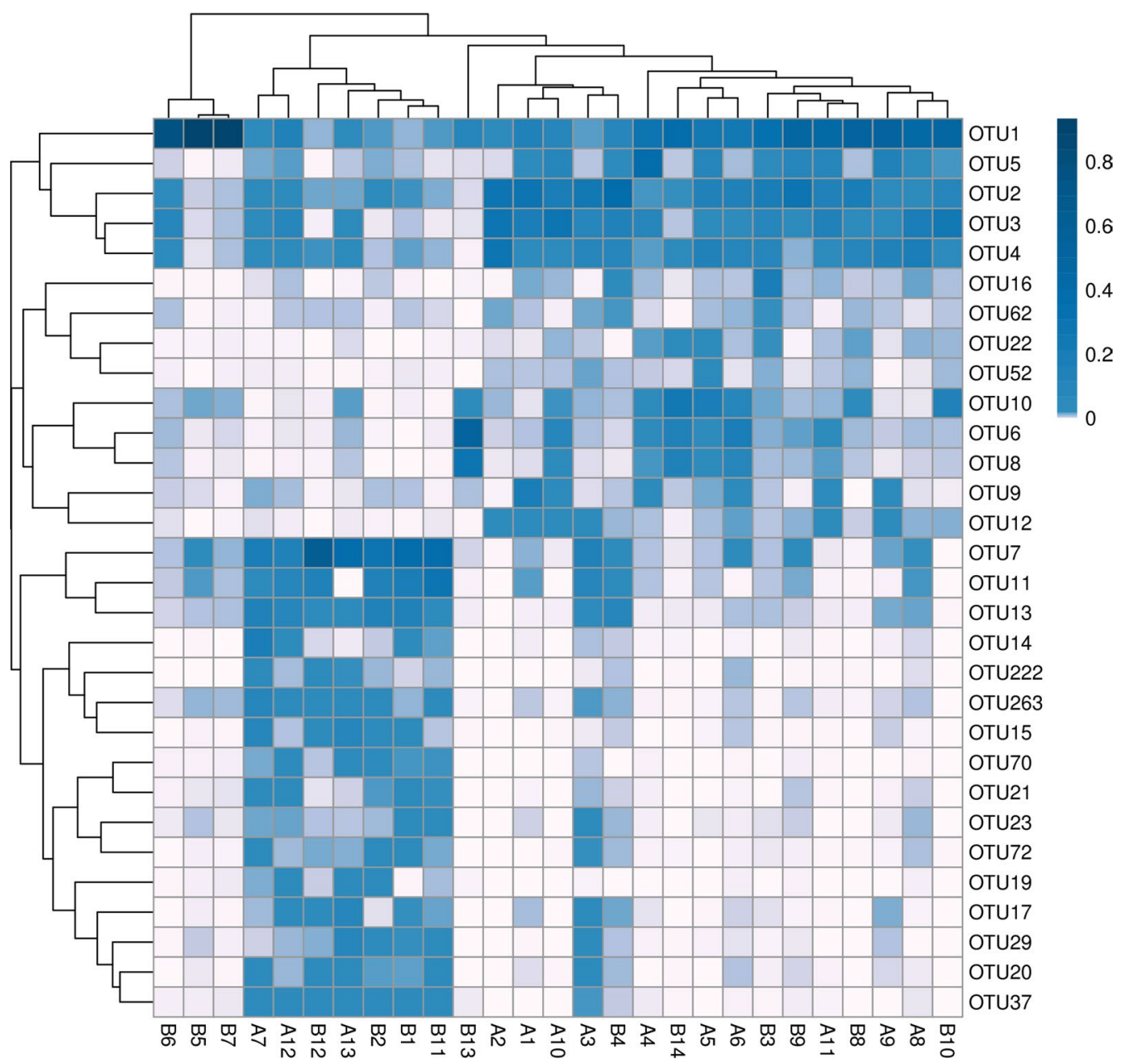

Fig. 1 Clustering analysis of the 30 most abundant OTUs in groups A and B. Hens in group A was fed conventional feed supplemented with a crude Astragalus (0.5\%) additive, while those in group B was fed only conventional feed

significant differences in the ACE, Chaol and Shannon indexed between two groups. The results indicated that species diversity was more abundant in group A than in the group B. The rarefaction curves of the microbiota of 27 samples were sufficiently large to estimate phenotype richness and microbial community diversity at a similarity threshold of $97 \%$ (Fig. 2). The rarefaction curves indicated that the sampling effort had sufficient sequence coverage to accurately describe the bacterial composition of each group.

\section{Microbial beta diversity analysis}

In a beta diversity analysis, the variations of the microbial community composition in 27 samples were shown in PCoA plots, with PC1 accounting for $43.12 \%$ of the total variation and $\mathrm{PC} 2$ accounting for $17.11 \%$ (Fig. 3). There were overlaps among four clusters and the microbial communities of samples A7, A12, A13, B1, B2, B11, and B12 were similar, as were the communities of samples A1, A2, A3, B4, and A10, while the other samples belonged to the same cluster.

Table 2 Diversity estimation of the 16S rRNA gene libraries of the 27 samples from the 16S rRNA sequences

\begin{tabular}{lllr}
\hline Group & ACE & Chao 1 & Shannon \\
\hline$A(n=13)$ & $245.0552 \pm 51.5936$ & $248.4307 \pm 55.2267$ & $4.0145 \pm 1.1526$ \\
$B(n=14)$ & $241.7484 \pm 54.5922$ & $247.0312 \pm 55.5076$ & $3.2576 \pm 1.4677$ \\
\hline
\end{tabular}

Group A = control; Group B = basal diet $+0.5 \%$ Astragalus

$a, b$ Different superscript letters in the same column indicate significant differences 


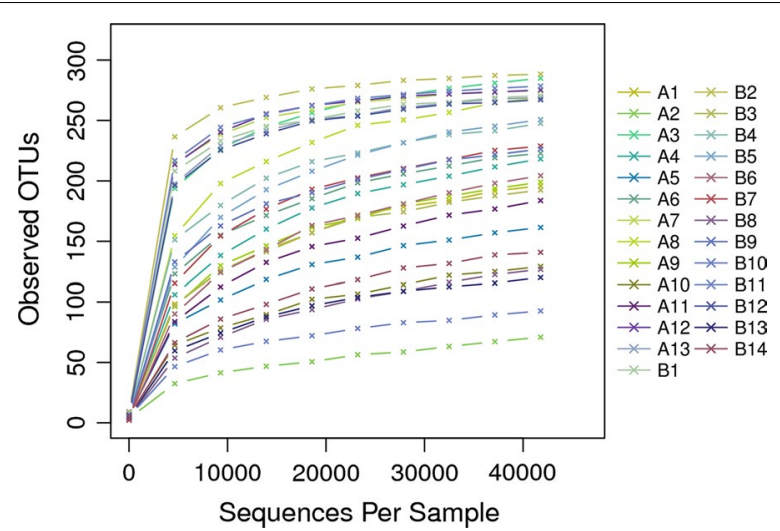

Fig. 2 Rarefaction curve sequences showing the complexities of the microbial communities in the fecal samples of 27 young hens. Samples A1-A13 were collected from hens fed conventional feed supplemented with a crude Astragalus (0.5\%) additive, while samples B1-B14 were collected from hens fed conventional feed

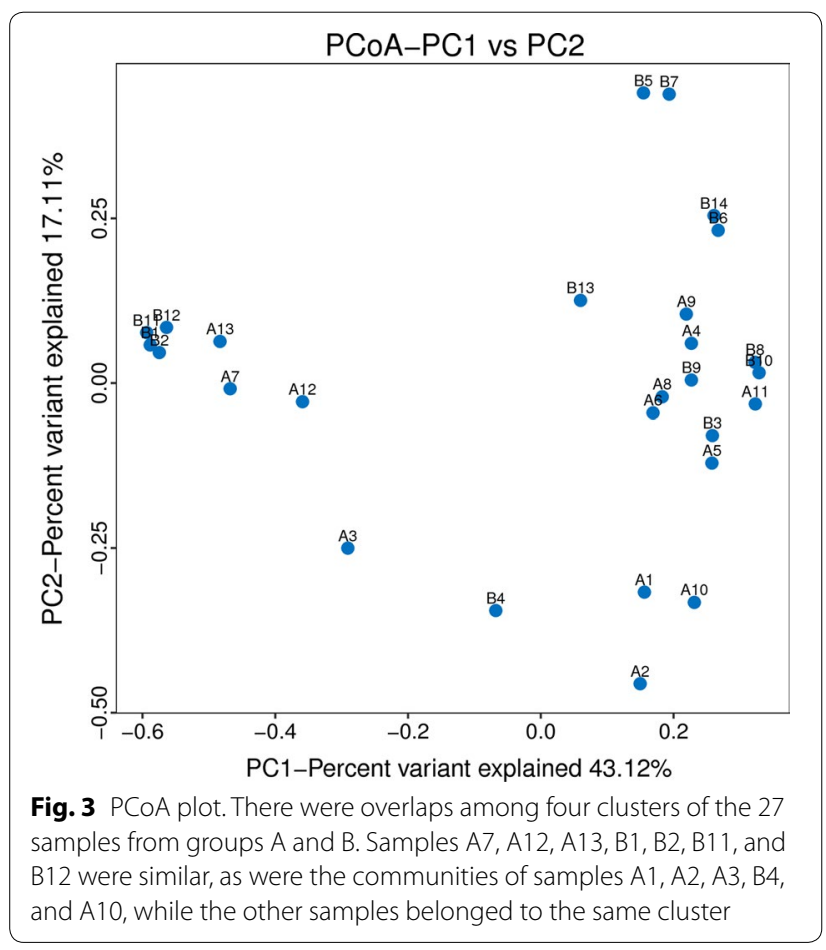

\section{Bacterial community composition}

At the phylum level, a total of 11 dominant phyla were identified in the two groups (Fig. 4a). Most of the sequences in group A were identified as Firmicutes (76.71\%), Bacteroidetes (13.50\%), and Proteobacteria (8.70\%) species, while Firmicutes (71.39\%), Bacteroidetes (17.60\%), and Proteobacteria (10.01\%) species were most abundant in group B (Fig. 4b).
As shown in Fig. 4c, d, at the genus level, the sequences from the 27 samples represented 31 dominate genera in total. Romboutsia (17.94\%), Lactobacillus (28.61\%), Bacteroides (10.65\%), and Enterococcus (10.51\%) were the most abundant taxa in group A, while Romboutsia (33.98\%), Lactobacillus (13.80\%), Bacteroides (15.07\%), and Enterococcus (4.35\%) were the most abundant in group B. The differences in the abundances of Lactobacillus were significant $(p<0.05)$.

\section{A community compositional heat map combined with a cluster analysis}

As shown in Fig. 5, the top 30 genera in terms of abundance were clustered and plotted using $\mathrm{R}$ software. The genera with higher abundances in the corresponding samples are shown in blue and those with lower abundances are shown in white. The populations of Lactobacillus and Romboutsia increased remarkably between groups A and B. Moreover, Romboutsia was more abundant in group B than group A. The reason for this difference may be partially attributed to the different $\mathrm{pH}$ of the feed fed to groups A and B (pH 4.96 vs. 4.71, respectively).

\section{Statistical analysis of taxonomic and functional profiles (STAMP)}

Differences between the two groups at the genus level were compared using STAMP (v2.1.3) software and the Welch's $t$-test. As shown in Fig. 6, the abundance of Lactobacillus was significantly $(p<0.05)$ greater in group A than group B. However, differences in the abundances of other genera were not significant.

\section{Discussion}

Although many studies have investigated the gut microbiota composition of poultry, very few have assessed the interactions between HMs and gut microbes. In recent years, the potential application of HMs in aquatic animals and rat models has been increasingly explored. A recent study showed that Yu-Ping-Feng (Jade screen) powder (Chinese parsnip root, Astragalus membranaceus and Atractylodes macrocephala Koidz) can regulate the intestinal microbiota of fish (Wu et al. 2018), the combination of Gancao-Gansui impacted the gut microbiota diversity of the rat (Yu et al. 2018), and Salvia miltiorrhiza Bge. modulated the microbiota imbalance in diabetic mice (Gu et al. 2017). Previous studies have shown that fermented Ginkgo leaves contributed to the microbial ecology in the gut of broiler chicks (Zhang et al. 2015), while fermented pine needles (Pinus ponderosa) improved antioxidant status and growth performance in broilers (Wu et al. 2015) and improved meat quality and lipid metabolism in broilers (Cao et al. 2012). The principle of HMs 

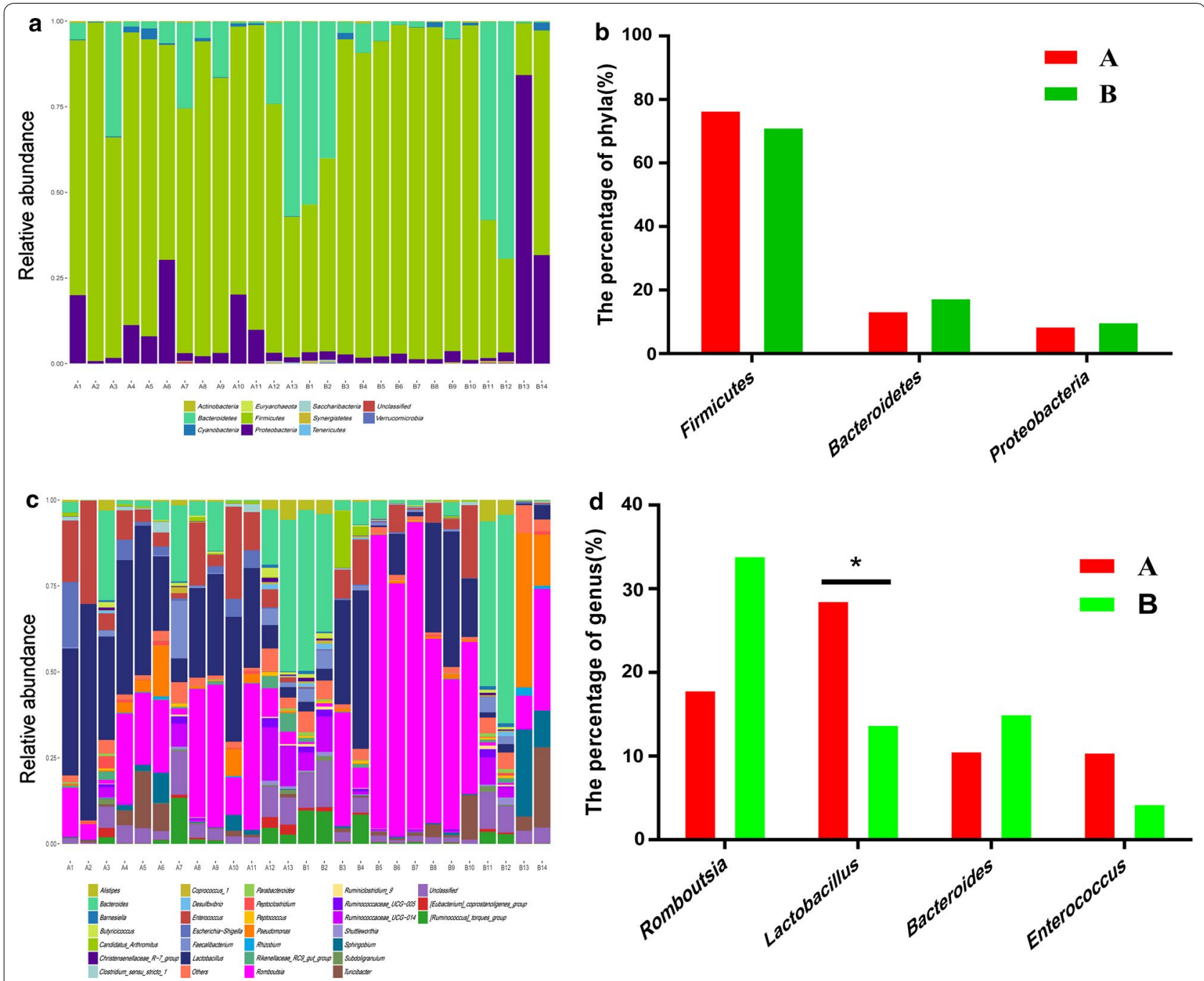

Fig. 4 Phylum-level and genus-level analyses of the 27 samples. a Overall fecal microbiota composition of the samples at the phylum level in groups A and B. b Comparison of the relative abundances of the three main bacterial phyla in groups A and B. c Overall fecal microbiota composition of the samples at the genus level in groups A and B. $\mathbf{d}$ Comparison of the relative abundances of the four main bacterial genus in groups $A$ and B. The relative abundances of Romboutsia, Lactobacillus, Bacteroides, and Enterococcus are shown on the $y$-axis. Boxes with one asterisk indicate significant differences between the two groups at $p<0.05$ using a $t$ test

supplementation of feed lies in maintaining or restoring the balance or enhancing the ability of immune defenses (Guo et al. 2004). Therefore, the aim of the present study was to compare the gut microbiota composition of young laying hens fed a diet supplemented with or without Astragalus supplement.

In this study, at the phylum level, Firmicutes, Bacteroidetes, and Proteobacteria were the most common phyla in the poultry fecal samples, which is consistent with earlier findings by Danzeisen et al. (2011) and Yeoman et al. (2012). Our results illustrate that Firmicutes were the dominant phylum ( $>50 \%$ ) in young laying hens, an observation that is consistent with previous reports (Mohd Shaufi et al. 2015; Danzeisen et al. 2011). Previous studies have also suggested that a lower abundance of Bacteroidetes spp. was associated with increased body weight (Arumugam et al. 2011; Ley et al. 2006). The results of the present study showed that the abundance of Bacteroidetes app. was lower in hens fed a diet supplemented with Astragalus, as compared with the control group, as Astragalus produces two kinds of polysaccharides that are common carbohydrates (Xu et al. 2008).

Numerous studies have also shown that Bacteroidetes aid in the host metabolism of polysaccharides, which improves nutrient utilization (Bäckhed et al. 2004) and host immunity (Hooper 2004; Stappenbek et al. 2002), as well as maintenance of the intestinal microecological balance (Hooper et al. 2001; Sears 2005). Animals are 


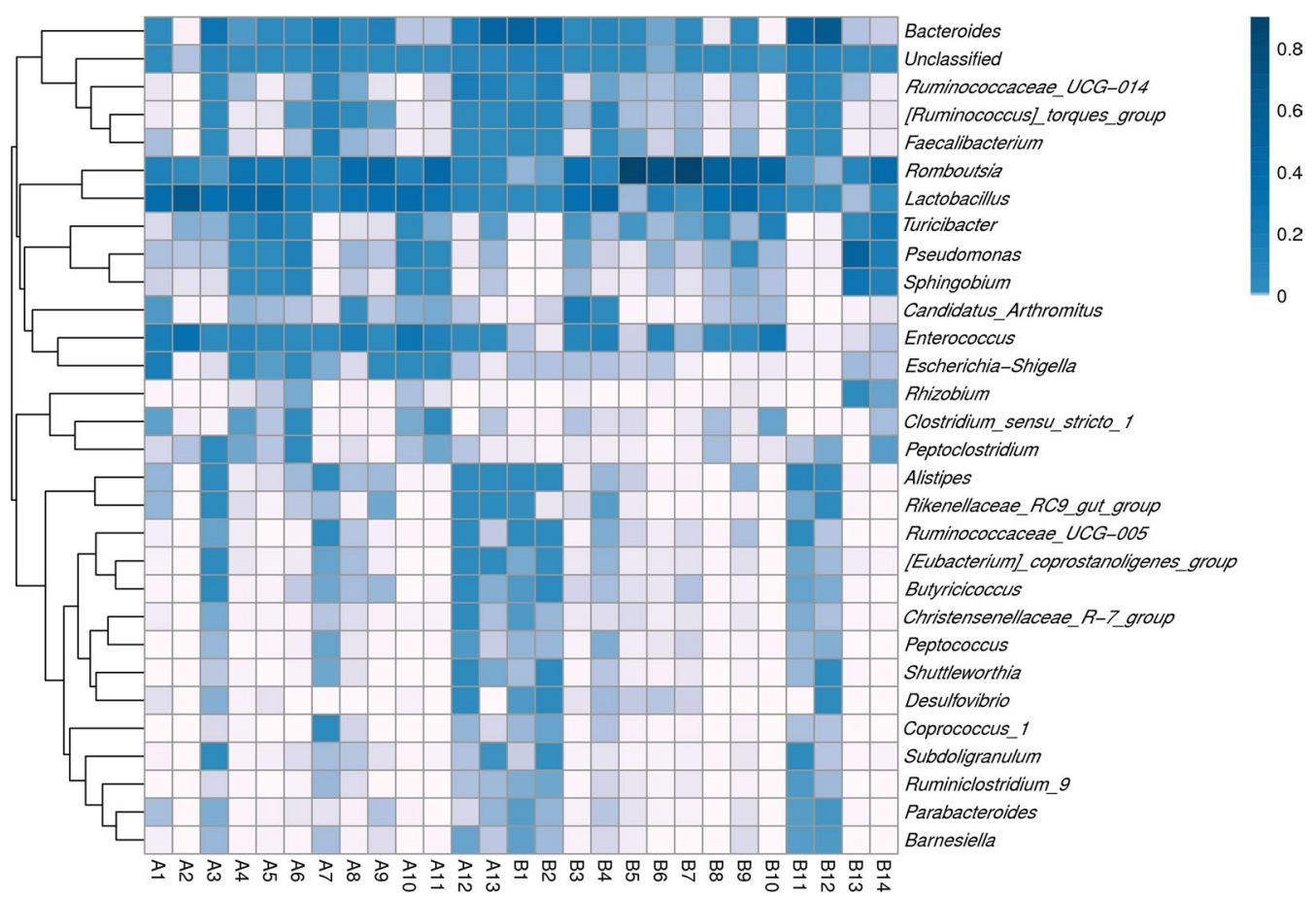

Fig. 5 Heat map analysis of the 27 samples. Heat map showing the abundances of the top 30 genera were clustered and plotted using R software. Blue represents genera with higher abundances in the corresponding sample, and white represents genera with lower abundances

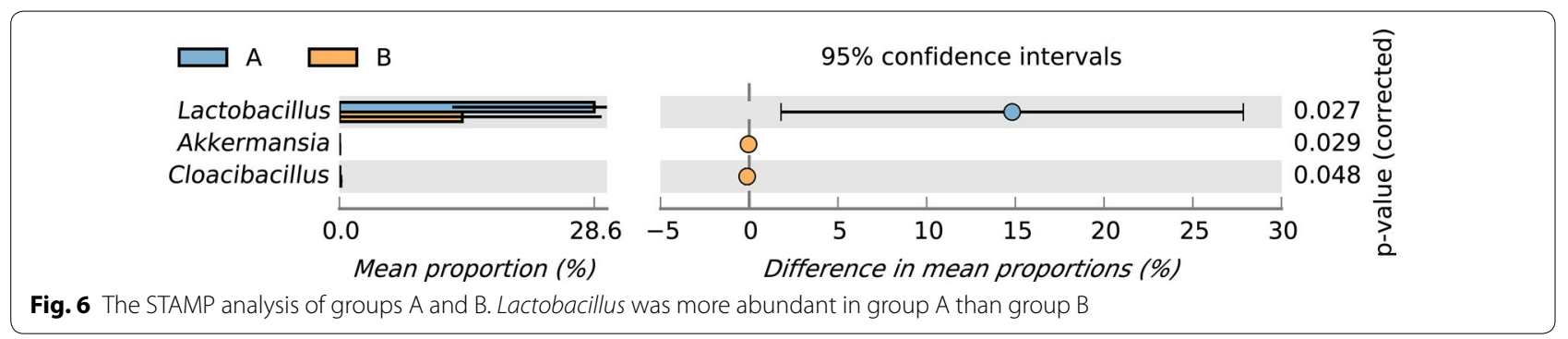

unable to digest and utilize complex polysaccharides in feedstuff in the absence of microbial fermentation (Vrize et al. 2010). In addition, our results differ from those of Singh et al. (2012) who showed that Proteobacteria was the most dominant phylum. The abundances of Proteobacteria, which include a wide range of pathogens, such as Escherichia, Salmonella, Helicobacter, and Vibrio spp., were slightly lower in group A than in group B, indicating that the abundance of opportunistic pathogens might decrease in response to supplementation with Astragalus. Therefore, the addition of Astragalus to feed may be beneficial for modulating the gut microbiota of young hens.

At the genus level, Romboutsia, Lactobacillus, Bacteroides, and Enterococcus were identified as the dominant species of the fecal microbiome. STAMP analysis results revealed that Lactobacillus was more abundant in group A than group B ( $28.61 \%$ vs. $13.80 \%$, respectively, $p<0.05)$, in agreement with the reports by Stanley et al. (2012) and Gong et al. (2007). Moreover, a recent study showed that the abundance of Lactobacillus was highly related to the feeding efficiency of the host (Yan et al. 2017). Lactobacillus is an important probiotic that promotes the gut health of both humans and animals via the production of various short-chain fatty acids. In addition, the results of a rodent study showed that non-digestible fermentable carbohydrates and fibers can enhance the growth of specific beneficial gut bacteria (Bindels et al. 2015). It is reported that feeding different metabolite combinations produced by Lactobacillus plantarum increased the abundance of fecal lactic acid bacteria and reduced the population of 
Enterobacteriaceae in the gut of laying hens (Loh et al. 2014). Therefore, feed supplementation with Astragalus results in an increase in the abundance of Lactobacillus, which is thought to be indicative of the health of hens. Moreover, in this study, the abundance of Bacteroides was lower in group A than group B. Bacteroides, which include the genera Bacteroides, Prevotella and Xylanibacter, are known to be efficient degraders of dietary fiber (Simpson and Campbell 2015). Bacteroides are thought to play fundamental roles in the breakdown of complex polysaccharides, suggesting that Bacteroides may be involved in the metabolism of Astragalus polysaccharides.

To the best of our knowledge, this is the first study of a novel Romboutsia genus (group A 17.94\%; group B 33.98\%) in fecal samples of hens, as members of the Romboutsia genus have never before been detected in the digestive tract of hens. Romboutsia spp. have been identified in the human gut (Ricaboni et al. 2016), lake sediment (Wang et al. 2015), and the rat gastrointestinal tract (Gerritsen et al. 2014). The results of the present study demonstrated that Astragalus supplementation enriched the abundance of Lactobacillus and reduced the abundance of Romboutsia spp. Therefore, we speculate there is a competitive exclusion relationship between the abundance of Romboutsia spp. and Lactobacillus genera, although well-designed experiments focusing on bacterial interactions are required to verify these results.

It is well know that Astragalus polysaccharides mainly consist of glucose and xylose. Romboutsia is able to utilize glucose, but not xylose (Wang et al. 2015). However, the effect of Romboutsia metabolism on the quantity of these monosaccharides remains unclear, thus further studies are warranted. In a future study, we plan to isolate and characterize the functions of Romboutsia spp.

Although fecal samples, instead of the cecal samples, were used to study the microbiome in this study, a previous study showed similarities in the microbial communities, but quantitative differences between cecum and fecal samples (Stanley et al. 2015). Moreover, as compared to cecal contents, fecal samples are easier to collect for analysis of the productivity, health, and wellbeing of chickens. However, sampling from cecum to other intestinal tract sections requires sacrificing of the hens. In contrast, fecal sampling allows repeated sampling over time and does not require sacrifice (Stanley et al. 2015). The results of the present study demonstrated that structural changes to the fecal microbiota are induced by Astragalus.

A previous study reported that HMs can alter the composition of the intestinal microbial community while being metabolized (Xu et al. 2017). Single HMs, HM formulas, and even individual compounds of HMs are capable of affecting the gut microbiota. Therefore, HMs may either promote or inhibit the gut microbiota. Similarly, some probiotics, such as Lactobacillus plantarum, have been shown to modulate fecal parameters in chickens (Gao et al. 2017), and Lactobacillus johnsonii has been shown to improve the gut microbiota in broiler chickens (Wang et al. 2017a). In our study, the addition of single Astragalus also modulated fecal microbiota. On the one hand, this might be due to the main bioactive constituents of Astragalus, such as polyphenols, which have anti-inflammatory and immunomodulatory effects that promote the abundances of some gut bacteria. On the other hand, it might result from interactions among gut microbiota after Astragalus feeding.

In conclusion, Astragalus is rich in fiber and polysaccharides that can be fermented and converted into shortchain fatty acids, which are beneficial to health and play a vital role in the modulation of fecal microbiota, although deciphering the underlying mechanism will require further research.

\section{Abbreviations}

HMs: herbal medicines; NGS: high-throughput, next-generation sequencing OTUs: operational taxonomic units; PCR: polymerase chain reaction; PCoA: principal coordinate analysis; STAMP: statistical analysis of taxonomic and functional profiles.

\section{Authors' contributions}

Conceived and designed the experiment: $\mathrm{HXQ}, \mathrm{CZB}$. Performed the experiments: LHZ, YZS, Data analysis: HTS. Wrote the paper: HXQ. All authors read and approved the final manuscript.

\section{Author details \\ ${ }^{1}$ College of Veterinary Medicine, Henan University of Animal Husbandry and Economy, Longzihu North road No.6, Zhengzhou 450046, Henan, People's Republic of China. ${ }^{2}$ Probiotics Bio-transformation Engineering Technology Research Center of Henan Province, Zhengzhou 450046, Henan, People's Republic of China. ${ }^{3}$ Key Laboratory of Probiotics Fermentation Traditional Chinese Medicine of Zhengzhou city, Zhengzhou 450046, Henan, People's Republic of China.}

\section{Acknowledgements}

Not applicable.

Competing interests

The authors declare that they have no competing interests.

\section{Availability of data and materials}

The data generated or analyzed during this study are included in this article.

\section{Consent for publication}

Not applicable.

\section{Ethics approval and consent to participate}

The protocols of the animal experiments were approved by the Laboratory Animal Management Committee of Henan University of Animal Husbandry and Economy and conducted in accordance with the Guidelines for the Care and Use of Experimental Animals.

\section{Funding}

This work was supported by the Henan Province Natural Science Planning Fund Projects [Grant No. 162300410128], by the Henan Province Science and Technology Open Cooperation Project [Grant No. 182106000042] and by the Veterinary Discipline Key Construction Project of Henan University of Animal Husbandry and Economy [Grant No. MXK2016102]. 


\section{Publisher's Note}

Springer Nature remains neutral with regard to jurisdictional claims in published maps and institutional affiliations.

Received: 3 January 2018 Accepted: 23 April 2018 Published online: 30 April 2018

\section{References}

Arumugam M, Raws J, Pelletier E, Le Paslier D, Yamada T, Mende DR, Fernandes GR, Tap J, Bruls T, Batto JM, Bertalan M, Borruel N, Casellas F, Fernandez L, Gautier L, Hansen T, Hattori M, Hayashi T, Kleerebezem M, Kurokawa K, Leclerc M, Levenez F, Manichanh C, Nielsen HB, Nielsen T, Pons N, Poulain J, Qin JJ, Sicheritz-Ponten T, Tims S, Torrents D, Ugarte E, Zoetendal EG, Wang J, Guarner F, Pedersen OM, de Vos W, Brunak S, Dore J, Consortium M, Weissenbach J, Dusko-Ehrlich S, Bork P (2011) Enterotype of the human gut microbiome. Nature 473(7346):174-180

Bäckhed F, Ding H, Wang T, Hooper LV, Koh GY, Nagy A, Semenkovich CF, Gordon Jl (2004) The gut microbiota as an environmental factor that regulates fat storage. Proc Natl Acad Sci USA 101(44):15718-15723

Bindels LB, Delzenne NM, Cani PD, Walter J (2015) Towards a more comprehensive concept for prebiotics. Nat Rev Gastroenterol Hepatol. 12:303-310

Borda-Molina D, Vital M, Sommerfeld V, Rodehutscord M, Camarinha-Silva A (2016) Insights into Broiler's gut microbiota fed with phosphorus, calcium, and phytase supplemented diets. Front Microbiol. 19(7):2033

Cao FL, Zhang XH, Yu WW, Zhao LG, Wang T (2012) Effect of feeding fermented Ginkgo biloba leaves on growth performance meat quality, and lipid metabolism broilers. Poult Sci 91:1210-1221

Chen F, Wen Q, Jiang J, Li HL, Tan YF, Li YH, Zeng NK (2016) Could the gut microbiota reconcile the oral bioavailability conundrum of traditional herbs? J Ethnopharmacol 179:253-264

Danzeisen JK, Kim HB, Isaacson RE, Tu ZJ, Johnson TJ (2011) Modulation of the chicken cecal microbiome and metagenome in response to anticoccidial and growth promoter treatment. PLOS ONE 6(11):e27949

Gao PF, Hou QC, Kwok Lai-Yu, Huo DX, Feng SZ, Zhang HP (2017) Effects of feeding Lactobacillus plantarum P-8 on the faecal microbiota of broiler chickens exposed to lincomycin. Sci Bull. 62(2):105-113

Gerritsen J, Fuentes S, Grievink W, Niftrik LV, Tindall BJ, Timmerman HM, Rijkers GT, Smidt H (2014) Characterization of Romboutsia ilealis gen. nov., sp. nov., isolated from the gastro-intestinal tract of a rat, and proposal for the reclassification of five closely related members of the genus Clostridium into the genera Romboutsia gen. nov., Intestinibacter gen. nov., Terrisporobacter gen. nov. and Asaccharospora gen. nov. Int J Syst Evol Microbiol 64:1600-1616

Gong J, Si W, Forster RJ, Huang R, Yu H, Yin Y, Yang C, Han Y (2007) 16S rRNA gene-based analysis of mucosa-associated bacterial community and phylogeny in the chicken gastrointestinal tracts: from crops to ceca. FEMS Microbiol Ecol 59(1):147-157

Gu JF, Su SL, Guo JM, Zhu Y, Zhao M, Duan JA (2017) The aerial parts of Salvia miltiorrhiza Bge. strengthen intestinal barrier and modulate gut microbiota imbalance in streptozocin-induced diabetic mice. J Funct Foods. 36:362-374

Guo FC, Williams BA, Kwakkel RP, Li HS, Li XP, Luo JY, Li WK, Verstegen MWA (2004) Effects of mushroom and herb polysaccharides, as alternatives for an antibiotic, on the cecal microbial ecosystem in broiler chickens. Poult Sci 83:175-182

Hill DA, Hoffmann C, Abt MG, Du Y, Kobuley D, Kirna TJ, Bushmanb FD, Artisa D (2010) Metagenomic analysis reveal antibiotic-induced temporal and spatial changes in intestinal microbiota with associated alterations in immune cell homeostasis. Mucosal Immunol 3(2):148-158

Hooper LV (2004) Bacterial contribution to mammalian gut development. Trends Microbiol 12(3):129-134

Hooper LV, Wong MH, Thelin A, Hansson L, Falk PG, Gordon JI (2001) Molecular analysis of commensal host-microbial relationship in the intestinal. Science 291(5505):881-884

Ibrahim LF, Marzouk MM, Hussein SR, Kauashty SA, Mahmoud K, Saleh NAM (2013) Flavonoid constituents and biological screening of Astragalus bombycinus Boiss. Nat Prod Res 27(4-5):386-393
Ismail AS, Behrendt CL, Hooper LV (2009) Reciprocal interactions between commensal bacteria and gamma delta intraepithelial lymphocytes during mucosal injury. J Immunol. 182(5):3047-3054

Kallon S, Li X, Ji J, Chen C, Xi Q, Chang S, Xue C, Ma J, Xie Q, Zhang Y (2013) Astragalus polysaccharide enhances immunity and inhibits H9N2 avian influenza virus in vitro and in vivo. J Anim Sci Biotechnol 4(1):22-37

Kim EJ, Yang KS (2005) Anti lipid peroxidative activity of Radix Astragali ceus. Yakhak Hoechi 49:11-19

Kim JH, Kim MH, Yang G, Huh Y, Kim SH, Yang WM (2013) Effects of topical application of Radix Astragalus on allergic dermatitis. J Immunopharmacol Immunotoxicol 235(1):151-156

Ley RE, Turnbaugh PJ, Klein S, Gordon JI (2006) Microbial ecology: human gut microbes associated with obesity. Nature 444(7122):1022-1023

Li SP, Zhao XJ, Wang JY (2009) Synergy of Astragalus polysaccharides and probiotics (Lactobaciilus and Bacillus cereus) on immunity and intestinal microbiota in chicks. Poult Sci 88(3):519-525

Li Y, Yao J, Han C, Yang J, Chaudhry MT, Wang S, Liu H, Yin Y (2016) Quercetin, inflammation and immunity. Nutrients 8(3):167

Loh TC, Choe DW, Foo HL, Sazili AQ, Bejo MH (2014) Effects of feeding different postbiotic metabolite combinations produced by Lactobacillus plantarum strains on egg quality and production performance, faecal parameters and plasma cholesterol in laying hens. BMC Vet Res 10:149-158

Lv YW, Hu W, Wang YL, Huang LF, He YB, Xie XZ (2011) Identification and determination of flavonoida in astragali radix by high performance liquid chromatography coupled with DAD and ESI-MS detection. Molecules 16(3):2293-2303

Mohd Shaufi MA, Sieo CC, Chong CW, Gan HM, Ho YW (2015) Deciphering chicken gut microbial dyamics based on high-throughput 16S rRNA metagenomics analyses. Gut Pathog 7:4

Park SH, Lee SI, Ricke SC (2016) Microbial populations in naked neck chicken ceca raised on pasture flock fed with commercial yeast cell wall prebiotics via an Illumina MiSeq Platform. PLoS ONE 11(3):e0151944

Qiao HX, Shi HT, Zhang Z, Jiang YL, Bian CZ (2017) Characteristics of Bacillus subtilis HNMY-13 and HNMY-15 strains in aflatoxin B1 degradation and Astragalus bio-transformation. Afr J Biotechnol 16(38):1882-1888

Qin Q, Niu J, Wang Z, Xu W, Qiao Z, Gu Y (2012) Astragalus membaranceus inhibits inflammation via phospho-p38 mitogen-activated protein kinase (MAPK) and nuclear factor (NF)-кB pathways in advanced glycation end product-stimulated macrophages. Int J Mol Sci 13:8379-8387

Qiu J (2007) Traditional medicine-a culture in the balance. Nature 448:126-128

Rastall RA, Gibson GR (2015) Recent development in prebiotics to selectively impact beneficial microbes and promote intestinal health. Curr opin biotechnol 32:42-46

Ricaboni D, Mailhe M, Khelaifia S, Raoult D, Million M (2016) Romboutsia timonensis, a new species isolated from human gut. New Microbes New Infec. 12:6-7

Sanpha K, Li XY, Ji J, Chen CY, Xi QY, Chang S, Xue C, Ma J, Xie Q, Zhang Y (2013) Astragalus polysaccharide enhances immunity and inhibits H9N2 avian influenza virus in vitro and in vivo. J Anim Sci Biotechnol 4(1):22-33

Sears CL (2005) A dynamic partnership: celebrating our gut flora. Anaerobe 11(5):247-251

Simpson HL, Campbell BJ (2015) Review article: dietary fibre-microbiota interactions. Aliment Pharmacol Ther 42:158-179

Singh KM, Shah T, Deshpande S, Jakhesara S, Koringa P, Rank DN, Joshi CG (2012) High through put 165 rRNA gene-based pyrosequencing analysis of the fecal microbiota of high FCR and low FCR broiler growers. Mol Biol Rep 39(12):10595-10602

Stanley D, Denman SE, Hughes RJ, Geier MS, Crowley TM, Chen H, Haring VR, Moore RJ (2012) Intestinal microbiota associated with differential feed conversion efficiency in chickens. Appl Microbiol Biotechnol 96(5):1361-1369

Stanley D, Geier MS, Chen HL, Hughes RJ, Moore RJ (2015) Comparison of fecal and cecal microbiotas reveals qualitative similarities but quantitative differences. BMC Microbiol 15:51

Stappenbek TS, Hooper LV, Gordon JI (2002) Development regulation of intestinal angiogenesis by indigenous microbes via Paneth cells. Proc Natl Acad Sci USA 99(24):15451-15455

Videnska P, Rahman MM, Faldynova M, BabakV, Matulova ME, Prukner-Radocic E, Krizek I, Smole-Mozina S, Kovac J, Szmolka A, Nagy B, Sedlar K, Cejkova D, Rychlik I (2014) Characterization of egg laying hen and broiler fecal 
microbota in poultry farms in Croatia, Czech republic, Hungary and Slovenia. Plos ONE 9(10):e110076

Vrize A, Holleman F, Zoetenal EG, de Vos WM, Hoekstra JB, Nieuwdorp M (2010) The environment within: how gut microbiota may influence metabolism and body composition. Diabetologia 53:606-613

Wang YW, Song JL, Zhai Y, Zhang C, Gerritsen J, Wang HM, Chen XL, Li YT, Zhao BQ, Zhao B, Ruan ZY (2015) Romboutsia sedimentorum sp. nov., isolated from an alkaline-saline lake sediment and emended description of the genus Romboutsia. Int J Syst Evol Microbiol 65:1193-1198

Wang HS, Ni XQ, Qing XD, Zeng D, Luo M, Liu L, Li GY, Pan KC, Jing B (2017a) Live probiotic Lactobacillus johnsonii BS15 promotes growth performance and lowers fat deposition by improving lipid metabolism, intestinal development, and gut microflora in broilers. Front Microbiol 8:1073-1087

Wang Y, Zhang RM, Li JY, Wu ZW, Yin WJ, Schwarz Stefam, Tyrrell JM, Zheng Y, Wang S, Shen Z, Liu Z, Liu J, Lei L, Li M, Zhang Q, Wu C, Zhang Q, Wu Y, Walsh TR, Shen J (2017b) Comprehensive resistome analysis reveals the prevalence of NDM and MCR-1 in Chinese poultry production. Nat Microbiol 2:16260-16267

Willing BP, Russell SL, Finalay BB (2011) Shifting the balance: antibiotic effects on host-microbiota mutualism. Nat Rev Microbiol 9(4):233-243

Wu QJ, Wang ZB, Wang GY, Li YX, Qi YX (2015) Effects of feed supplemented with fermented pine needles (Pinus ponderosa) on growth performance and antioxidant status in broilers. Poult Sci 94:1138-1144

Wu ZB, Gatesoupe FJ, Li TT, Wang XH, Zhang QQ, Feng DY, Feng YQ, Chen H, Li AH (2018) Significant improvement of intestinal microbiota of gibel carp (Carassius auratus gibelio) after traditional Chinese medicine feeding. J Appl Microbiol 3:829-841

Xiao HB, Krucker M, Albert K, Liang XM (2004) Determination and identification of isoflavonoids in Radix astragali by matrix solid-phase dispersion extraction and high-performance liquid chromatography with photodiode array and mass spectrometric detection. J Chromatogr A 1-2:117-124

Xie W, Gu D, Li J, Cui K, Zhang Y (2011) Effect and action mechanisms of berberine and Rhizoma coptidis on gut microbes and obesity in high-fat die-fed C57BL/6J mice. PLoS ONE 6(9):e24520
Xu DJ, Xia Q, Wang JJ, Wang PP (2008) Molecular weight and monosaccharide composition of Astragalus polysaccharides. Molecules 13:2408-2415

Xu J, Lian F, Zhao L, Zhao Y, Chen X, Zhang X, Guo Y, Zhang C, Zhou Q, Xue Z, Pang X, Zhao L, Tong X (2015) Structural modulation of gut microbiota during alleviation of type 2 diabetes with a Chinese herbal formula. ISME J 9:552-562

Xu YH, Yang HX, Zhang LL, Su TH, Shi DH, Xiao HD, Tian Y (2016) High-throughput sequencing technology to reveal the composition and function of cecal microbiota in Dgu chicken. BMC Microbiol 16:259-268

Xu J, Chen HB, Li SL (2017) Understanding the molecular mechanisms of the interplay between Herbal medicine and gut microbiota. Med Res Rev 37(5):1-46

Yan W, Sun CJ, Yuan JW, Yang N (2017) Gut metagenomic analysis reveals prominent role of lactobacillus and cecal microbiota in chicken feed efficiency. Sci Rep 7:45308

Yang Y, Chen G, Yang Q, Ye J, Cai X, Tsering P, Cheng X, Hu C, Zhang S, Cao P (2017) Gut microbiota drives the attenuation of dextran sulphate sodiuminduces colitis by Huangqin decoction. Oncotarget 8(30):48863-48874

Yeoman CJ, Chia N, Jeraldo P, Sipos M, Goldenfeld ND, White BA (2012) The microbiome of the chicken gastrointestinal tract. Anim Health Res Rev 13(1):89-99

Yu YG, Guo JM, Tao WW, Liu P, Shang EX, Zhu ZH, Fan XH, Shen J, Hua YQ, Zhu KY, Tang YP, Duan JA (2018) Gancao-Gansui combination impacts gut microbiota diversity and related metabolic functions. J Ethnopharmacol 214:71-82

Zhang X, Sun Z, Cao F, Ahmad H, Yang X, Zhao L, Wang T (2015) Effects of dietary supplementation with fermented Ginkgo leaves on antioxidant capacity, intestinal morphology and microbial ecology in broiler chicks. Br Poult Sci 56(3):370-380

\section{Submit your manuscript to a SpringerOpen ${ }^{\odot}$ journal and benefit from:}

- Convenient online submission

- Rigorous peer review

- Open access: articles freely available online

- High visibility within the field

- Retaining the copyright to your article

Submit your next manuscript at $\boldsymbol{\nabla}$ springeropen.com 